Mayra Alexandra Carillo-Rodríguez; Mercedes Isabel Ruiz-Morales; Jorge Santiago Santamaria-Serrano; Elsa María Caín-Yuquilema

http://dx.doi.org/10.35381/r.k.v5i1.888

\title{
La dramatización para el perfeccionamiento de la destreza oral en el idioma inglés
}

\section{Dramatization for the improvement of oral skills in the English language}

Mayra Alexandra Carillo-Rodríguez

mayra.carillo@espoch.edu.ec

Escuela Superior Politécnica de Chimborazo, Riobamba

Ecuador

https://orcid.org/0000-0003-4162-7793

Mercedes Isabel Ruiz-Morales

mercedes.ruiz@espoch.edu.ec

Escuela Superior Politécnica de Chimborazo, Riobamba

Ecuador

https://orcid.org/0000-0002-9784-5848

Jorge Santiago Santamaria-Serrano

santiago.santamaria@espoch.edu.ec

Escuela Superior Politécnica de Chimborazo, Riobamba

Ecuador

https://orcid.org/0000-0003-2910-0489

Elsa María Caín-Yuquilema

ecain@institutos.gob.ec

Instituto Superior Pedagógico Intercultural Bilingüe Jaime Roldós Aguilera, Colta

Ecuador

https://orcid.org/0000-0001-9148-4685

Recibido: 21 de mayo de 2020

Revisado: 19 de junio de 2020

Aprobado: 25 de julio de 2020

Publicado: 01 de agosto de 2020 
Mayra Alexandra Carillo-Rodríguez; Mercedes Isabel Ruiz-Morales; Jorge Santiago Santamaria-Serrano; Elsa María Caín-Yuquilema

\title{
RESUMEN
}

El objetivo de investigación el cual se basó en determinar la relación de la dramatización como recurso didáctico para el perfeccionamiento de la destreza oral del idioma inglés en estudiantes de la Escuela Superior Politécnica de Chimborazo (ESPOCH). Se realizó desde el enfoque cuantitativo, correspondiendo a un tipo descriptivo en correlación de Pearson, con diseño no experimental transversal. La correlación es positiva en ,365* y significativa bilateral en el nivel 0,05 lo cual implica que existe relación entre la variable dramatización (DRM) y la destreza oral (DO), aunado que se acepta la hipótesis afirmativa y se rechaza la nula. La dramatización influye positivamente en el aprendizaje del inglés, específicamente en la destreza oral, por cuanto involucra los diversos canales o estilos de aprendizaje como es el visual, auditivo, kinestésico, propiciando el trabajo cooperativo y colaborativo.

Descriptores: Enseñanza de idiomas; laboratorio de idiomas; teatro; experimento educacional. (Palabras tomadas del Tesauro UNESCO).

\begin{abstract}
The research objective which was based on determining the relationship of dramatization as a didactic resource for the improvement of oral skills of the English language in students of the Polytechnic School of Chimborazo (ESPOCH). It was carried out from the quantitative approach, corresponding to a descriptive type in Pearson's correlation, with a non-experimental cross-sectional design. The correlation is positive at $.365{ }^{*}$ and bilaterally significant at the 0.05 level, which implies that there is a relationship between the dramatization variable (DRM) and oral dexterity (OD), in addition to accepting the affirmative hypothesis and rejecting the null. Dramatization has a positive influence on the learning of English, specifically on oral skills, since it involves the various channels or learning styles such as visual, auditory, and kinesthetic, promoting cooperative and collaborative work.
\end{abstract}

Descriptors: Language instruction; language laboratories; theatre; educational experiments. (Words taken from the UNESCO Thesaurus). 


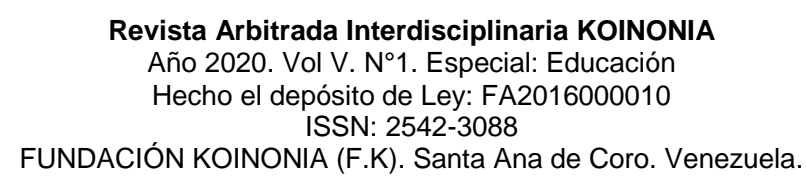

Mayra Alexandra Carillo-Rodríguez; Mercedes Isabel Ruiz-Morales; Jorge Santiago Santamaria-Serrano; Elsa María Caín-Yuquilema

\section{INTRODUCCIÓN}

La pedagogía que es ciencia y arte a la vez, ciencia en cuanto se afianza en estudios que así la avalan, pero arte en cuanto la práctica diaria es la que realmente logra hacer que la persona se desarrolle como pedagoga en toda la extensión de la palabra y transmitir así de una manera efectiva un determinado conocimiento o simplemente una información, conjugan el binomio que el educador debe cultivar para evitar caer en rutinas que le conlleven al desánimo del acto pedagógico que desde luego repercute en las personas a las cuales debe transferir la enseñanza requerida en un momento dado (Abreu, et al., 2017). Como el arte de enseñar, brinda el acceso a experimentar alternativas que contribuyan a su evolución con el propósito de brindar nuevas técnicas y estrategias a las personas que ejecutan una acción pedagógica, por cuanto así podrán brindar un acto pedagógico favorable al servicio de las personas que se encuentran en un aula de clases o en un determinado ambiente de aprendizaje (Ramos, et al., 2017).

Las estrategias didácticas son de suma importancia a la hora de propiciar un proceso de enseñanza - aprendizaje favorable para los actores involucrados en el mismo (UruetaVélez, 2019), las estrategias deben ser diseñadas y utilizadas por el docente o facilitador pensando en la interacción con una audiencia heterogénea, en la cual se pueden encontrar estudiantes con una percepción visual, auditiva, kinestésica, más desarrollada que otros y por ende aplicando la premisa de los empiristas que afirman que el conocimiento se produce por los sentidos (experiencia) del individuo, se debe combinar las estrategias didácticas con el propósito de generar un conocimiento significativo en los entes involucrados en el proceso de enseñanza - aprendizaje (De-Armas, M, \& Rodríguez, 2015).

Por lo tanto, el estudiante de acuerdo al nivel perceptivo que posea será quien asumirá el control de la situación de estudio, la estrategia viene a representar una especie de guía motivacional que el estudiante asumirá si va acorde con la percepción cognitiva que éste posee, de allí que aplicar una estrategia acorde en el momento apropiado, al público apropiado, es casi seguro que conduzca al éxito de las personas involucradas en el 


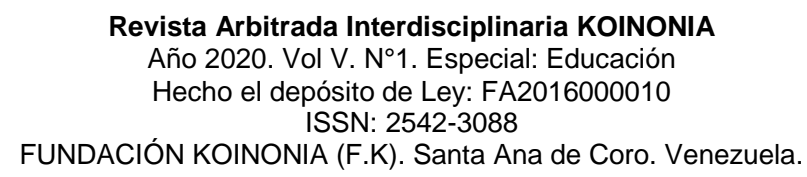

Mayra Alexandra Carillo-Rodríguez; Mercedes Isabel Ruiz-Morales; Jorge Santiago Santamaria-Serrano; Elsa María Caín-Yuquilema

proceso educativo (Hidalgo-Cajo, et al. 2017), tanto el docente como el estudiante se ven beneficiados cuando esto ocurre de manera favorable, por cuanto el docente habrá cumplido con su meta que es transmitir del mejor modo posible una determinada enseñanza y el estudiante se beneficia en cuanto puede absorber en todo su potencial esta enseñanza para adquirir y construir un aprendizaje favorable que en muchos casos será significativo (Vallejo-Valdivieso, et al., 2019).

En este sentido, una de las formas pedagogía de generar aprendizaje es a través del teatro y dramatización (Cruz-Colmenero, et al., 2013), desde donde los docentes pueden diseñar, experimentar, estrategias didácticas con la finalidad de estimular la motivación en los estudiantes en aprender sobre un determinado tema o mejorar una competencia para su crecimiento integral en función de un rendimiento académico favorable en consecución de una educación de calidad e inclusiva. Así en la enseñanza de idiomas, especialmente sí esta es una lengua extranjera, podría resultar que la dramatización logre mejorar la expresión oral, mediante la interpretación de personajes con los cuales según su nivel cognitivo se identifiquen (Roca-Castro, et al., 2019).

Actualmente, el inglés es reconocido como una lengua franca mundial, lo que significa que las personas de todo el mundo están aprendiendo inglés como su primer, segundo o idioma extranjero. El número de hablantes de este idioma aumenta constantemente por cuanto juega un papel muy importante en la sociedad globalizada. Por lo tanto, hablar este idioma se ha convertido en una habilidad personal de vital importancia para ser competitivos en el siglo XXI, en base a esta necesidad, en Ecuador se ha promovido el proceso de enseñanza-aprendizaje del idioma inglés en primaria, secundaria e incuso en la educación superior (Ramírez-Lozada, et al., 2018).

Enseñar efectivamente el inglés como segunda lengua en el sistema educativo de modo efectivo y asertivo, es un desafío exigente que requiere que los maestros usen actividades dinámicas e interactivas para lograr resultados significativos, es necesario implementar un método para desarrollar esta importante habilidad comunicativa (Ricoy, \& Álvarez-Pérez, 2016). Una alternativa significativa para lograr estos objetivos es la 


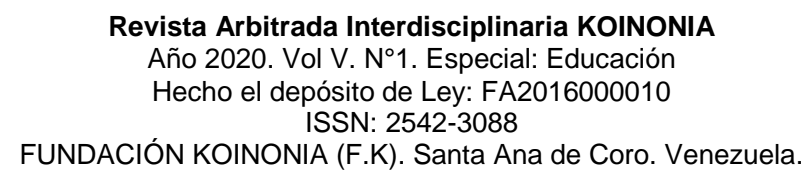

Mayra Alexandra Carillo-Rodríguez; Mercedes Isabel Ruiz-Morales; Jorge Santiago Santamaria-Serrano; Elsa María Caín-Yuquilema

dramatización, además, los juegos de roles, la simulación y la mímica son parte de las actividades teatrales que se centran en las habilidades del habla para mejorar a los estudiantes en una práctica significativa de las competencias comunicativas (SalazarBlanco, et al., 2015).

Además, es indispensable desarrollar habilidades de habla en los estudiantes, con la finalidad de alcanzar una oralidad asertiva en proporción de comunicarse efectivamente, esto por cuanto siendo el Ecuador un país con potencial turístico, económico de intercambio, así como la sociedad del conocimiento de la era digital, hacen necesario la comunicación en inglés, posibilitando a los jóvenes contar con nuevas alternativas de estudios y crecimiento personal, oportunidades que pueden potenciar a la nación en las próximas décadas a su desarrollo integral (Mora-Pisco, et al., 2018).

Para tal fin se tiene en cuenta la dramatización como estrategia pedagógica de enseñanza muy valiosa que involucra a los estudiantes en un proceso de aprendizaje activo, involucrándose el juego de roles con la intención de cooperar a los maestros en la generación de una atmósfera positiva y agradable en el aula, propiciando motivación para que los estudiantes aprendan el idioma de modo objetivo. La forma más efectiva de desarrollar las habilidades de habla del idioma inglés es facilitando la actividad y participación de los estudiantes en la práctica, dado que la técnica ayuda al desarrollo de habilidades orales, fomenta el crecimiento de la fluidez, la pronunciación, el aprendizaje cooperativo, la autoconfianza, así como la conciencia cultural. Los juegos de roles pueden tener un impacto positivo en los estudiantes porque los alientan a usar su imaginación, creatividad e innovar, proyectando personas con aptitudes de emprendimiento y liderazgo social (Rico-Yate, et al., 2016).

En función de lo planteado, la Escuela Superior Politécnica de Chimborazo (ESPOCH), ubicada en Riobamba - Chimborazo - Ecuador, incluye el inglés como asignatura, siendo un requisito tener un dominio del idioma extranjero del nivel B1 del CERF (Marco Común Europeo de Referencia para las lenguas: aprendizaje, enseñanza, evaluación), para completar los estudios, desde este contexto, la institución ha incluido la materia en el 
Mayra Alexandra Carillo-Rodríguez; Mercedes Isabel Ruiz-Morales; Jorge Santiago Santamaria-Serrano; Elsa María Caín-Yuquilema

pensum y los docentes buscan alternativas metodológicas para cumplir este objetivo. La institución está preocupada por promover un buen ambiente para que los estudiantes aprendan un idioma extranjero, por cuanto debe certificarse en el dominio del idioma inglés en el nivel B1 para permitirles graduarse, siendo desde esta visión una oportunidad aplicar un programa de aprendizaje basado en la dramatización como estrategia didáctica para incentivar la expresión oral en estudiantes universitarios.

Desde lo planteado se formula el objetivo de investigación el cual se basó en determinar la relación de la dramatización como recurso didáctico para el perfeccionamiento de la destreza oral del idioma inglés en estudiantes de la Escuela Superior Politécnica de Chimborazo (ESPOCH).

\section{MÉTODO}

La investigación se realizó desde el enfoque cuantitativo, correspondiendo a un tipo descriptivo en correlación de Pearson, con diseño no experimental transversal, siendo la observación la técnica de recolección de información, aplicándose dos instrumentos tipo lista de cotejo, en donde se anotaban las intervenciones de los estudiantes en cuanto a la dramatización y en otro su destreza oral, así se procedió a estructurar en frecuencias y luego calcular el coeficiente de correlación. La muestra poblacional estuvo conformada por 30 estudiantes de inglés de nivel B1 de la Escuela Superior Politécnica de Chimborazo (ESPOCH). 
Mayra Alexandra Carillo-Rodríguez; Mercedes Isabel Ruiz-Morales; Jorge Santiago Santamaria-Serrano; Elsa María Caín-Yuquilema

\section{RESULTADOS}

\section{Cuadro 1}

Correlación entre dramatización y destreza oral

DRM DO

\begin{tabular}{llrr}
\hline DRM & Correlación de Pearson & 1 &, $365^{*}$ \\
& Sig. (bilateral) & 0,047 \\
& N & 30 & 30 \\
DO & Correlación de Pearson &, $365^{\star}$ & 1 \\
& Sig. (bilateral) & 0,047 & \\
& N & 30 & 30 \\
& & \\
\hline${ }^{*}$ La correlación es significativa en el nivel 0,05 (bilateral).
\end{tabular}

La correlación es positiva en, $365^{\star}$ y significativa bilateral en el nivel 0,05 lo cual implica que existe relación entre la variable dramatización (DRM) y la destreza oral (DO), aunado que se acepta la hipótesis afirmativa y se rechaza la nula.

\section{Prueba de hipótesis}

H1: La relación de la dramatización como recurso didáctico para el perfeccionamiento de la destreza oral del idioma inglés es significativa en estudiantes de la Escuela Superior Politécnica de Chimborazo (ESPOCH).

H0: La relación de la dramatización como recurso didáctico para el perfeccionamiento de la destreza oral del idioma inglés no es significativa en estudiantes de la Escuela Superior Politécnica de Chimborazo (ESPOCH). 


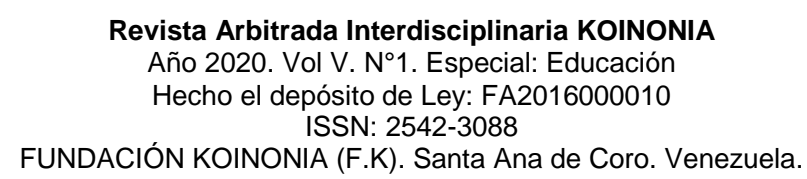

Mayra Alexandra Carillo-Rodríguez; Mercedes Isabel Ruiz-Morales; Jorge Santiago Santamaria-Serrano; Elsa María Caín-Yuquilema

\section{DISCUSIÓN}

Al ser significativa la relación de la dramatización como recurso didáctico para el perfeccionamiento de la destreza oral del idioma inglés, concuerda con los resultados mostrados por (Salas, 2016), quien indica que la dramatización o el teatro aplicado a la clase de inglés contribuye a fomentar un mejor aprendizaje, involucrando los estilos visual, auditivo, kinestésico, constituyéndose en una alternativa viable y concreta para ser ejecutada con regularidad por los docentes en las aulas de clases, por cuanto el estudiante tiene la oportunidad de crear un ambiente de empatía con sus compañeros, sumado a la posibilidad de promover su motivación al logro académico.

En este sentido (Vieites, 2017), plantea considerar la pedagogía teatral como un recurso para acceder a los diversos niveles educativos, por cuanto esta permite desde una vertiente holística formar al participante en la consecución de un aprendizaje basado en la experiencia y construcción de saberes desde el trabajo en equipo, así mismo (LatapíEscalante, 2018), destaca la influencia del teatro sobre la emocionalidad, lo cual se constituye en una arista favorable para promover un asertivo aprendizaje en el estudiante, contribuyendo a la conformación de la autorregulación emocional.

La educación emocional a juicio de (Del-Pino-Peña \& Aguilar-Fernández, 2013), contribuye a fomentar el liderazgo estudiantil, así implícitamente no solo se trabaja en la mejora de aspectos pedagógicos para el abordaje correcto de la oralidad en el inglés, cooperando con a educación formal en su rol socializador de los actores educativos, compaginándose con la posibilidad de contar con ciudadanos proactivos en función de aportar en la generación de un mejor contexto social, por cuanto el sistema educativo ha proveído la formación axiológica de un ser crítico - reflexivo.

El pensador critico desde la postura de (Prieto-Galindo, 2018), debe generar un proceso lógico de razonamiento objetivo, proyectando derrumbar mitos sobre sí mismo, fundamentado desde la razón como eje de acción para el abordaje de la realidad social donde se socializa el estudiante habitualmente, de ese modo una pedagogía teatral contribuye a reflexionar desde el arte en qué aspectos de la destreza oral en el inglés, 


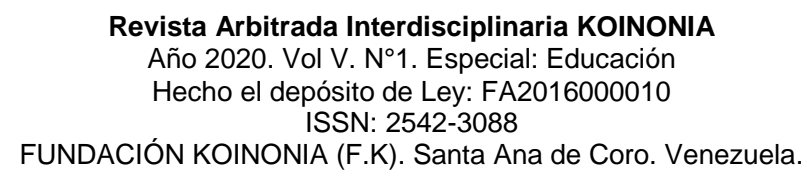

Mayra Alexandra Carillo-Rodríguez; Mercedes Isabel Ruiz-Morales; Jorge Santiago Santamaria-Serrano; Elsa María Caín-Yuquilema

reamente se dominan, en donde se debe mejorar, como mejorar, aceptando la crítica del docente y compañeros como parte de la auto reflexión, permitiendo desechar o aceptar las premisas según sea el acontecimiento a analizar, así la emocionalidad podrá conjugarse en equilibrio del crecimiento adecuado del estudiante para superar los retos que implica aprender efectivamente una lengua extranjera.

En cuanto a la enseñanza del inglés, (Clouet, 2010) considera que el Marco Común Europeo de Referencia para las lenguas: aprendizaje, enseñanza, evaluación, ha sido uno de los métodos más relevantes, siendo necesario enseñar desde una visión ecléctica de la educación, siendo así que la dramatización se configura como una genuina alternativa para alcanzar un efectivo aprendizaje, lo cual es confirmado por la revisión bibliográfica de (Juárez-Díaz \& Perales-Escudero, 2019), donde se destaca que las experiencias significativas de los estudiantes se encuentran relacionadas con los profesores, la tecnología y el método de enseñanza, siendo importante tener en cuenta la formación permanente del docente para que este pueda aplicar los métodos y tecnologías en función de propiciar un efectivo proceso educativo, sin dejar de lado el manejo emocional para generar la empatía necesaria para construir desde un punto común, desde lo colaborativo como acción edificante del conocimiento (RomeroGuarquila, et al., 2020).

\section{CONCLUSIÓN}

La dramatización influye positivamente en el aprendizaje del inglés, específicamente en la destreza oral, por cuanto involucra los diversos canales o estilos de aprendizaje como es el visual, auditivo, kinestésico, propiciando el trabajo cooperativo y colaborativo con fines de construir el conocimiento desde una óptica donde se promueve la motivación, autorreflexión, visión axiológica de la realidad social, aunado a la creatividad, innovación, improvisación, al tener que construir guiones, asumir el papel de un determinado personaje, lo cual puede ser considerado divertido, afianzándose en la experiencia del estudiante y así reconocer lo significativo de lo aprendido. 
Mayra Alexandra Carillo-Rodríguez; Mercedes Isabel Ruiz-Morales; Jorge Santiago Santamaria-Serrano; Elsa María Caín-Yuquilema

\section{FINANCIAMIENTO}

No monetario

\section{AGRADECIMIENTO}

A los estudiantes de la Escuela Superior Politécnica de Chimborazo; por el apoyo en el desarrollo de la investigación.

\section{REFERENCIAS CONSULTADAS}

Romero-Guarquila, L., García-Herrera, D., Ávila-Mediavilla, C., \& Erazo-Álvarez, J. (2020). Aprendizaje colaborativo para la motivación del aprendizaje de inglés. [Collaborative learning for the motivation of learning English]. EPISTEME KOINONIA, 3(6), 273-290. http://dx.doi.org/10.35381/e.k.v3i6.825

Juárez-Díaz, C, \& Perales-Escudero, M. (2019). Experiencias en el aprendizaje del inglés en la educación superior. [Experiences in learning English in higher education]. Lenguaje, 47(2), 358378. https://dx.doi.org/10.25100/lenguaje.v47i2.6734

Clouet, R. (2010). El enfoque del marco común europeo de referencia para las lenguas: unas reflexiones sobre su puesta en práctica en las facultades de traducción e interpretación en España. [The approach of the common european framework of reference for languages: some considerations concerning its putting into practice in translation and interpreting faculties in Spain]. RLA. Revista de lingüística teórica y aplicada, 48(2), 71-92. https://dx.doi.org/10.4067/S0718$\underline{48832010000200004}$

Prieto-Galindo, F. (2018). El pensamiento crítico y autoconocimiento. [El pensamiento crítico y autoconocimiento]. Revista de filosofía, 74, 173191. https://dx.doi.org/10.4067/S0718-43602018000100173

Del-Pino-Peña, R, \& Aguilar-Fernández, M. (2013). La inteligencia emocional como una herramienta de la gestión educativa para el liderazgo estudiantil. [Emotional Intelligence as an Educational Management Tool to Enhance Student Leadership from EconomicAdministrative and Technical Areas]. Cuadernos de Administración, 29(50),132-141. 
Mayra Alexandra Carillo-Rodríguez; Mercedes Isabel Ruiz-Morales; Jorge Santiago Santamaria-Serrano; Elsa María Caín-Yuquilema

Latapí-Escalante, P. (2018). El teatro histórico como ámbito educativo. Análisis de cuatro obras para conmemorar el triunfo de la República en Querétaro, México. [The historical theater as an educational field. Analysis of four works to commemorate the triumph of the Republic in Querétaro, Mexico]. Historia $Y$ MEMORIA, (17), 185-217. https://doi.org/10.19053/20275137.n17.2018.7438

Abreu, O, Gallegos, M, Jácome, J, \& Martínez, R. (2017). La Didáctica: Epistemología y Definición en la Facultad de Ciencias Administrativas y Económicas de la Universidad Técnica del Norte del Ecuador. [Didactics: Epistemology and Definition in the Faculty of Management and Economics at the Technical University of Northern Ecuador]. Formación universitaria, 10(3), 8192. https://dx.doi.org/10.4067/S0718-50062017000300009

Cruz-Colmenero, V, \& Caballero-García, P, \& Ruiz-Tendero, G. (2013). La dramatización como recurso didáctico para el desarrollo emocional. Un estudio en la etapa de educación primaria. [Dramatization as a didactic resource for emotional development. A study in the primary education stage]. Revista de Investigación Educativa, 31(2), 393-410.

De-Armas, M, \& Rodríguez, M. (2015). Estudio de los estilos de aprendizaje de estudiantes y profesores de la Facultad de Ingeniería de la Universidad Central de Venezuela. [Study of the learning styles of students and professors of the Faculty of Engineering of the Central University of Venezuela]. Revista de la Facultad de Ingeniería Universidad Central de Venezuela, 30(3), 17-26.

Hidalgo-Cajo, B, Mayacela-Alulema, Á, \& Hidalgo-Cajo, I. (2017). Estrategias didácticas para potenciar el aprendizaje de Farmacología clínica. [Didactic strategies to improve the learning of clinical Pharmacology]. Revista Habanera de Ciencias Médicas, 16(3), 439-453.

Mora-Pisco, L, Díaz-Rodríguez, N, \& Vergara-Cevallos, D. (2018). El turismo en la matriz productiva de Ecuador: resultados y retos actuales. [Tourism in the productive matrix of Ecuador: Current results and challenges]. Revista Universidad y Sociedad, 10(5), 255-262.

Ramírez-Lozada, H., Perlaza-Rodríguez, M. K., Sosa-Daza, E. P., \& Bautista-Salazar, F. S. (2018). Los Métodos de Enseñanza del inglés en la Preparación Idiomática Requerida por la Universidad del Siglo XXI. [The Methods of Teaching English in the Language Preparation Required by the University of the XXI Century]. Revista Científica Hallazgos21, 3(Suplemento especial), 1-8. 
Mayra Alexandra Carillo-Rodríguez; Mercedes Isabel Ruiz-Morales; Jorge Santiago Santamaria-Serrano; Elsa María Caín-Yuquilema

Ramos, J, Rhea, B, Pla, R, \& Abreu, O. (2017). La Pedagogía como Ciencia para el Tratamiento de los Contenidos Generales del Proceso Educativo y la Formación de Valores. [Pedagogy as a Science for the Treatment of the General Contents of the Educational Process and the Formation of Values]. Formación universitaria, 10(6), 77-86. https://dx.doi.org/10.4067/S0718-50062017000600009

Ricoy, M, \& Álvarez-Pérez, S. (2016). La enseñanza del inglés en la educación básica de personas jóvenes y adultas. [Teaching English in the Basic Education of Youth and Adults]. Revista mexicana de investigación educativa, 21(69), 385-409.

Rico-Yate, J, Ramírez-Montoya, M, \& Montiel-Bautista, S. (2016). Desarrollo de la competencia oral del inglés mediante recursos educativos abiertos. [Development of oral competence through the use of Open Educational Resources]. Apertura (Guadalajara, Jal.), 8(1), 1-15.

Roca-Castro, Y., \& Véliz-Robles, F. (2019). Importancia de las estrategias didácticas para mejorar el tratamiento de la gramática en el uso del idioma inglés en los estudiantes. Caso: Colegio Nacional "Abdón Calderón”. Ecuador. [Importance of didactic strategies to improve the treatment of grammar in the use of the English language in students. Case: "Abdón Calderón" National School. Ecuador]. CIENCIAMATRIA, 6(10), 182-203. https://doi.org/10.35381/cm.v6i10.123

Salas, J. (2016). El uso del teatro en la clase de inglés como estrategia didáctica. [The use of theater in the English class as a didactic strategy]. InterSedes, 17(36), 155209. https://dx.doi.org/10.15517/isucr.v17i36.27467

Salazar-Blanco, O, Echeverri-Restrepo, C, \& Díaz-Hernández, D. (2015). Reflexiones sobre la competencia comunicativa en los pregrados de las áreas de salud de universidades colombianas. [Reflections on communicative competence in the undergraduate health areas of Colombian universities]. FEM: Revista de la Fundación Educación Médica, 18(3), 211-217. https://dx.doi.org/10.4321/S2014$\underline{98322015000400010}$

Urueta-Vélez, L. (2019). Estrategias de enseñanza y el uso de las tecnologías de información y comunicación en las instituciones educativas departamentales en el Municipio Zona Bananera - Colombia. [Teaching strategies and the use of information and communication technologies in departmental educational institutions in the Municipality of Zona Bananera - Colombia]. Revista Arbitrada Interdisciplinaria Koinonía, 4(7), 185-201. http://dx.doi.org/10.35381/r.k.v4i7.200 
Revista Arbitrada Interdisciplinaria KOINONIA

Año 2020. Vol V. N¹. Especial: Educación

Hecho el depósito de Ley: FA2016000010

ISSN: 2542-3088

FUNDACIÓN KOINONIA (F.K). Santa Ana de Coro. Venezuela.

Mayra Alexandra Carillo-Rodríguez; Mercedes Isabel Ruiz-Morales; Jorge Santiago Santamaria-Serrano; Elsa María Caín-Yuquilema

Vallejo-Valdivieso, P., Zambrano-Pincay, G., Vallejo-Pilligua, P., \& Bravo-Cedeño, G. (2019). Estructuras mentales en la construcción de aprendizaje significativo. [Mental structures in the construction of meaningful learning]. CIENCIAMATRIA, 5(8), 228-241. https://doi.org/10.35381/cm.v5i8.257

Vieites, M. (2017). La Pedagogía Teatral como Ciencia de la Educación Teatral. [Theatre Pedagogy as the Science of Theatrical Education]. Educação \& Realidade, 42(4), 1521-1544. Epub August 07, 2017.https://dx.doi.org/10.1590/2175-623662918

C2020 por los autores. Este artículo es de acceso abierto y distribuido según los términos y condiciones de la licencia Creative Commons Atribución-NoComercial-Compartirlgual 4.0 Internacional (CC BY-NC-SA 4.0)

(https://creativecommons.org/licenses/by-nc-sa/4.0/). 